\title{
A Trimethylsilylamine-Acyl Fluoride Amide Bond Forming Proto- col for Weakly Nucleophilic Amines that is Amenable to the Paral- lel Synthesis of Di(hetero)arylamides
}

\author{
Maryam Zamiri \\ David S. Grierson* \\ Faculty of Pharmaceutical Sciences, The University of \\ British Columbia, 2405 Westbrook Mall, Vancouver, \\ V6T 1Z3, Canada \\ dgrierso@mail.ubc.ca
}

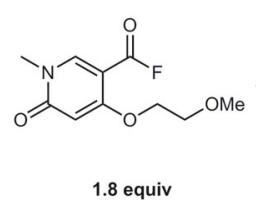

Received: 30.06 .2016

Accepted after revision: 31.08.2016

Published online: 29.09 .2016

DOI: 10.1055/s-0036-1588603; Art ID: ss-2016-n0421-op

Abstract The reaction of a 2-pyridinone-based acid fluoride with the N-TMS derivatives of different weakly nucleophilic heteroaryl/arylamines in acetonitrile containing catalytic fluoride ion provides a clean, efficient and simple means to access a diverse range of polar di(hetero)arylamide structures. This amide bond forming protocol is readily amenable to the parallel synthesis of compound libraries.

Key words amide coupling, di(hetero)arylamide, acid fluoride, heteroarylamine, $N$-TMS activation, parallel synthesis

The screening of compound libraries has repeatedly demonstrated its effectiveness as a research tool in drug discovery. In particular, when mechanistic and/or structural details for the therapeutic target are lacking, compound library screening is one of very few options possible to identify new and/or groundbreaking avenues for drug development. In our laboratory, parallel synthesis is used to connect and functionalize novel scaffolds into libraries of bioactive molecules containing different architectures. In particular, amide bond formation was used to generate molecules that possess a di(hetero)arylamide (DHA) substructure. The screening of a 256-membered library containing 119 DHA compounds identified four novel and related molecules 1-4 (Figure 1) that block HIV-1 replication by a mechanism involving perturbation of the alternative splicing events leading to production of key HIV regulatory proteins. $^{1 \mathrm{a}, \mathrm{b}}$

For the construction of the amide bond, a wide range of peptide (amide) bond forming reagents and conditions are available. ${ }^{2}$ However, in our library project, where electrondeficient (weakly nucleophilic) heterocyclic amines were engaged, ${ }^{3}$ the yield of the DHA product in reactions using
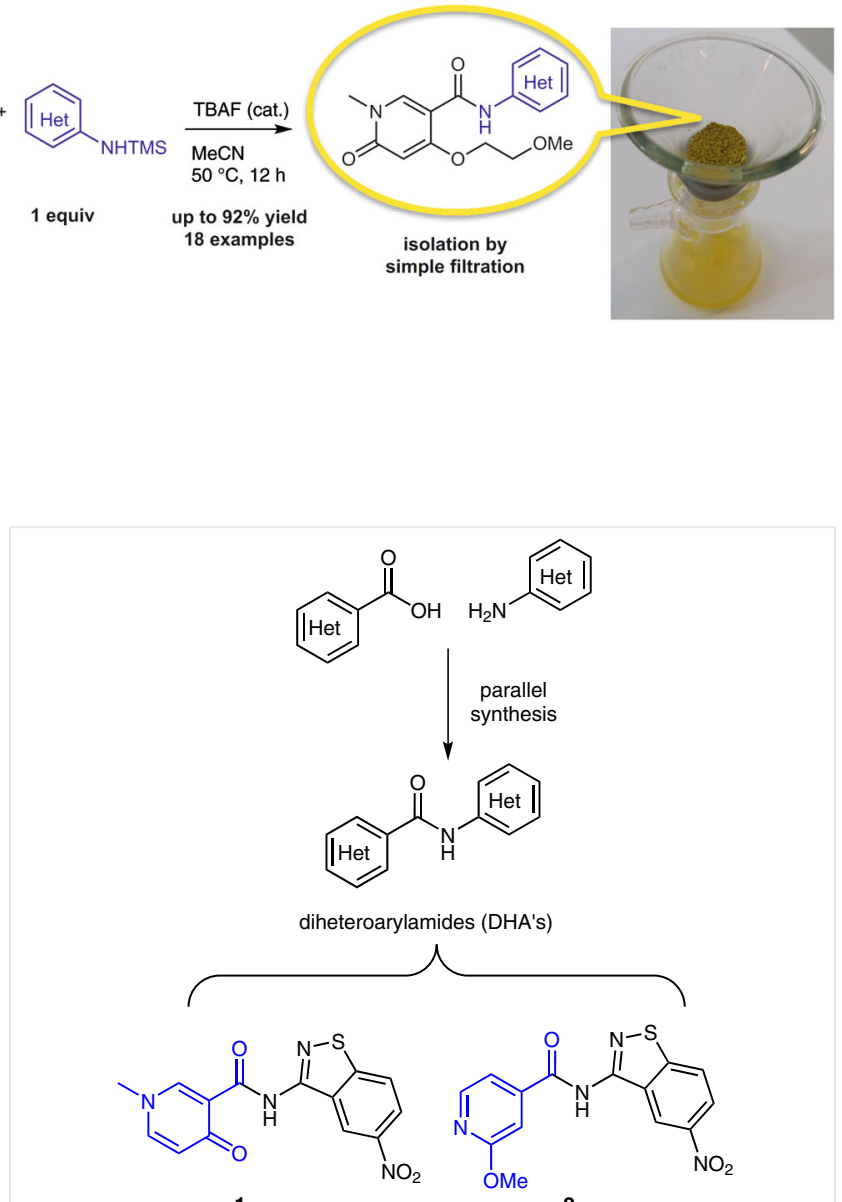<smiles>COCCOc1ccncc1C(=O)Nc1nsc2ccc([N+](=O)[O-])cc12</smiles>

3<smiles>COCCOc1cc(=O)n(C)cc1C(=O)Nc1nsc2ccc([N+](=O)[O-])cc12</smiles>

Figure 1 IDC16 (indole compound 16) mimics: di(hetero)arylamidetype anti-HIV compounds $\mathbf{1 - 4}$

the acid chloride method and different peptide coupling reagents rarely exceeded $10-30 \%$. In fact, the formation of complex mixtures of polar compounds was generally observed, complicating workup and isolation/purification of the desired product. To advance the anti-HIV project, and other applications for the DHA library, it became important to develop a new amide synthesis protocol. 
In this report, we describe the development of such a protocol for amide bond construction that is efficient and amenable to the parallel synthesis of novel di(hetero)arylamides. The procedure is based on the reaction of readily available $N$-trimethylsilylamines with acid fluorides in the presence of catalytic fluoride ion. Formally, the fluoride ion reacts with the silylated amine to form a 'hypervalent' silicon species, ${ }^{4}$ which readily decomposes to produce volatile TMSF and an amine anion intermediate, which is higher in energy and more nucleophilic/reactive than the corresponding free amine. Although often stable to water, and less reactive than acid chlorides toward alcohols, acid fluorides react efficiently with amines to give the expected amide products. ${ }^{5,6}$ An additional attractive feature of the use of acid fluorides and silylated amines as the coupling partners is that these heterocyclic components are nonpolar relative to their amine/acid precursors and to the amide products that are generated. Consequently, by choosing a reaction solvent in which the polar di(hetero)arylamide product selectively precipitates, its isolation is reduced to a simple filtration. For development of the protocol, the acid fluoride derivative of 2-pyridinone-5-carboxylic acid (6) was used as the acid component, as beyond the discovery of compound $\mathbf{4}^{1}$ (Figure 1 ) relatively little development of this scaffold in amide synthesis has been described. ${ }^{7}$

Carboxylic acid 6 was prepared (Scheme 1) by 04-alkylation of 2-pyridinone ester $5^{1,7}$ with 1 -bromo-2-methoxyethane (DMF, $\mathrm{Cs}_{2} \mathrm{CO}_{3}$ ) and subsequent hydrolysis of the ester group using LiOH in THF- $\mathrm{H}_{2} \mathrm{O}(2: 1)$. Conversion of 6 to acid fluoride 7 was best achieved through reaction with fluoro- $N, N, N^{\prime}, N^{\prime}$-tetramethylformamidinium hexafluorophosphate $(\mathrm{TFFH})^{8}$ and cesium fluoride $(\mathrm{CsF})$ in anhydrous $\mathrm{MeCN}$ at room temperature for 5 hours. The presence of added fluoride ion was mandatory in order to avoid com- peting formation of the symmetrical anhydride $8{ }^{9}$ Compound 7 was isolated pure as a colorless solid (90\%) by washing the crude product mixture with hexane to remove the tetramethylurea formed, and then taking the solid material up in dichloromethane, filtering to remove the excess $\mathrm{CsF}$, and concentrating to dryness. The structure of $\mathbf{7}$ was confirmed by the presence of a peak at $25.43 \mathrm{ppm}$ in the ${ }^{19} \mathrm{~F}$ NMR spectrum, and by the up-field shift $(\Delta \delta 0.14 \mathrm{ppm})$ from $\delta=8.34$ for the peak for $\mathrm{H}-6$ in the ${ }^{1} \mathrm{H}$ NMR spectrum. As expected, acid fluoride 7 was much more stable than the corresponding (and corrosive) acid chloride derivative, and could be conveniently stored for periods exceeding one month in an airtight container at $4{ }^{\circ} \mathrm{C}$. In fact, when dissolved in dichloromethane and washed with ice water, it was recovered in greater than $90 \%$ yield (purity check by ${ }^{1} \mathrm{H}$ NMR spectroscopy).

The diversity set of 18 trimethylsilylated heteroaryl/arylamines 9a-r (Table 1) used in this study was prepared by reacting $0.5 \mathrm{mmol}$ of the requisite amine in neat trimethylsilyl cyanide (TMSCN) $(0.5 \mathrm{~mL})$ at $70{ }^{\circ} \mathrm{C}$ (30 to $180 \mathrm{~min}$ ) (Table 1$).{ }^{10}$ The advancement of each reaction was monitored by ${ }^{1} \mathrm{H}$ NMR spectroscopy $\left(\mathrm{CDCl}_{3}\right)$. Interestingly, the time required to observe complete dissolution of the amine, that is, an indicator of complete N-silylation, appeared to be a function of both the reactivity of the amine and its solubility. Indeed, the duration of reaction required did not follow a clear linear relationship to the electron density on the exocyclic amine nitrogen (as calculated using Spartan software ${ }^{11}$ ) (see calculated $\mathrm{e}^{-}$-density values in Table 1). With the exception of TMS-amines $\mathbf{9 m}$ and $\mathbf{9 p}$, the polar amine substrates dissolved in TMSCN as the reaction progressed. As the only by-product of the reaction was volatile $\mathrm{HCN}$, it and the residual TMSCN were removed under vacuum.

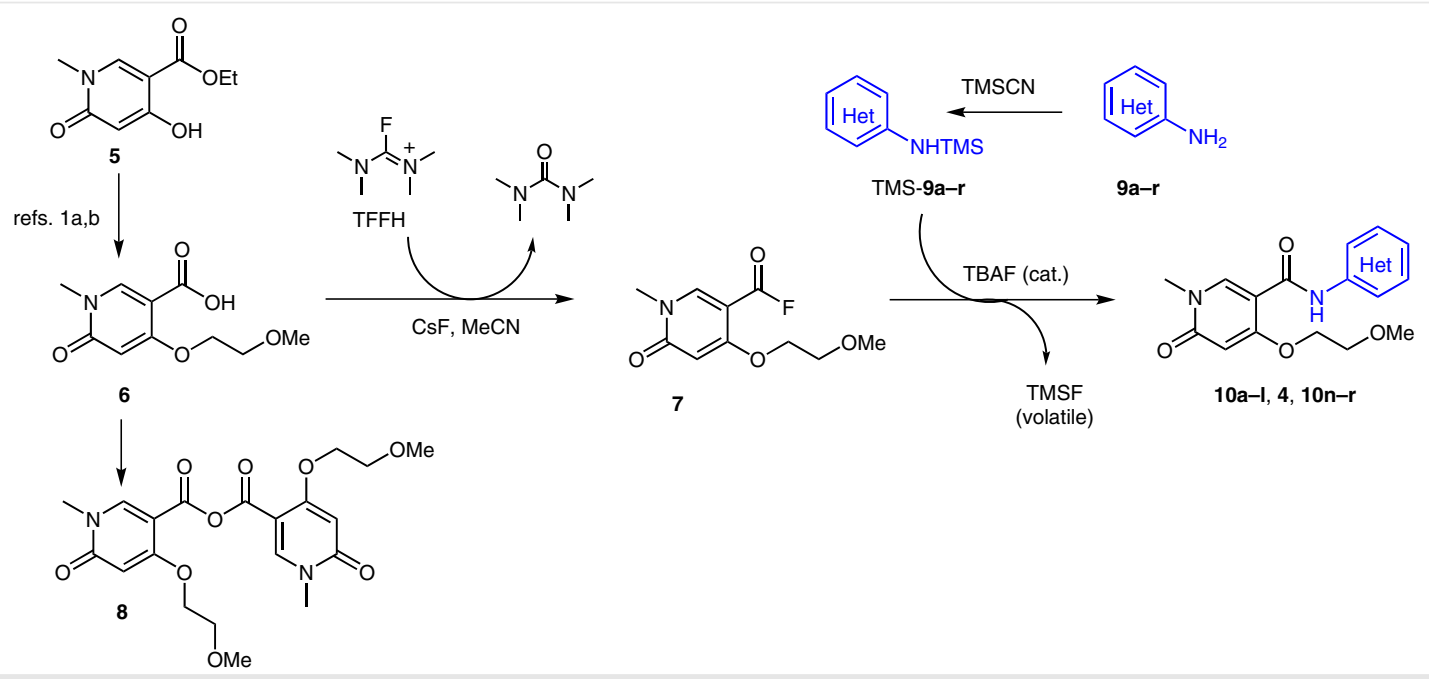

Scheme 1 Preparation of acyl fluoride 7 and its reaction with TMS-9a-r 
Synthesis

M. Zamiri, D. S. Grierson

Paper

Table 1 Synthesis of Di(hetero)arylamides $\mathbf{4}$ and $\mathbf{1 0}$ from TMS-Amines $\mathbf{9 a - r}$

\begin{tabular}{|c|c|c|c|c|}
\hline & TMS-amine $9^{a}$ & Electron density & N-Silylation time & Amide $^{\mathrm{a}, \mathrm{b}}$ (yield, \%) \\
\hline TMS-9a & & 140.1 & $7(91 \%)^{c}$ & $\mathbf{1 0 a}(78)$ \\
\hline TMS-9 $\mathbf{b}^{12}$ & & 132.2 & 30 & $10 \mathrm{~b}(7)$ \\
\hline TMS-9c & & 120 & 30 & $10 c(27)$ \\
\hline TMS-9d & & 119.5 & 15 & $\operatorname{10d}(34)$ \\
\hline TMS-9e & & 115 & 30 & $10 \mathbf{e}(53)$ \\
\hline TMS-9f ${ }^{13 a-d}$ & $T$ & 112.8 & 30 & $10 f(55)$ \\
\hline TMS-9 $\mathbf{g}^{14}$ & & 105.9 & 180 & $\log (89)$ \\
\hline TMS-9h $\mathbf{h}^{13 a}$ & & 100.5 & 90 & $10 \mathrm{~h}(42)$ \\
\hline TMS-9i & & 98 & 120 & $10 \mathbf{i}(62)$ \\
\hline TMS-9j & & 97.8 & 30 & $\mathbf{1 0 j}(71)$ \\
\hline TMS-9k & & 96 & $180(96 \%)^{c}$ & $10 \mathbf{k}(85)$ \\
\hline TMS-9|15 & & 92.9 & 30 & $101(87)$ \\
\hline TMS-9m ${ }^{1 b}$ & & 88.8 & 30 & $4(60)^{1 \mathrm{a,b}}$ \\
\hline TMS-9n & & 88.7 & 30 & 10n (92) \\
\hline TMS-9o ${ }^{13 a}$ & & 80.4 & 180 & $100(81)$ \\
\hline
\end{tabular}


Table 1 (continued)

\begin{tabular}{lllll}
\hline TMS-9p & Electron density & N-Silylation time & Amide \\
\hline a,b (yield, \%)
\end{tabular}

a All known compounds are cited by literature references.

${ }^{\mathrm{b}}$ Amide formation: TMS-amine $(0.5 \mathrm{mmol})+$ acid fluoride $(0.89 \mathrm{mmol})$ in $\mathrm{MeCN}(5 \mathrm{~mL}), 50{ }^{\circ} \mathrm{C}$, overnight, (isolated yield).

c Percent conversion to TMS-amine.

As alluded to already, reactions where the acid chloride derivative of carboxylic acid $\mathbf{6}$ (and the related compound containing a 4-OMe substituent ${ }^{1}$ ) was coupled to amines 9 using different solvents (DMF, $\mathrm{CH}_{2} \mathrm{Cl}_{2}$, THF) and bases $\left(\mathrm{Et}_{3} \mathrm{~N}\right.$, pyridine, $\mathrm{K}_{2} \mathrm{CO}_{3}$ ) at room temperature, or with heating, resulted in incomplete conversion to the desired amide products. Under these conditions, the product mixture contained unreacted amine/acid, as well as copious amounts of unidentified polar material. This, plus the fact that the product mixtures were poorly soluble, complicated purification and limited applicability to synthesis or parallel synthesis. In contrast, the reaction of the four (nonsilylated) amines 9c, 9g, 91, and 9n with excess of acid fluoride $\mathbf{7}$ in acetonitrile at $50{ }^{\circ} \mathrm{C}$ was remarkably efficient. Under these conditions the product precipitated from the medium as the reaction progressed, and was contaminated with much smaller quantities (up to 15\%) of residual amine. Encouraged by this result, the reaction of TMS-9n with an excess (1.8 equiv) of acid fluoride 7 was evaluated. It was observed that any residual amine that may not have reacted remained in solution, as amide 10n was isolated pure after filtration and drying in $47 \%$ yield. Further, a significant improvement in product yield (47\% to $92 \%$ ) was achieved by addition of a catalytic amount of TBAF ( $2 \mathrm{~mol} \%$ ) at the outset, to initiate reaction of fluoride ion with silicon and formation of the putative amine anion. The protocol that was subsequently employed for the synthesis of the new DHA molecules involved adding acid fluoride $7(0.89 \mathrm{mmol})$ in acetonitrile $(5 \mathrm{~mL})$ to the requisite TMS-amine TMS-9a-r $(0.5 \mathrm{mmol})$ (prepared immediately before the reaction). TBAF was then added, and the resulting mixture was heated at $50{ }^{\circ} \mathrm{C}$ overnight. After cooling, the precipitated material was collected by suction filtration and dried. As product purity, and not quantity, was the principle criterion for these reactions, efforts were generally not made to improve the amide product yields beyond those indicated in Table 1 . In this context, it was interesting to observe that the yield for the preparation of amide 10a from the electron-rich amine TMS-9a was comparable to that for the preparation of 10r from the electron-deficient amine TMS-9r. In fact, the initial yield for 10a was $38 \%$, but could be brought up to $78 \%$ by washing with a mixture of $\mathrm{MeCN} / \mathrm{Et}_{2} \mathrm{O}$. This reflected the fact that amide 10a is more soluble in MeCN than amide 10r. The relatively lower yields for the isolation of amides 10b-d also reflects their significant solubility in MeCN. For our purposes, ${ }^{1} \mathrm{H}$ NMR spectroscopy was used to determine product purity. In the large majority of cases, no impurities were detected, and in the few cases where the presence of the amine component persisted, only trace amounts were detectable (purity $>95 \%$ ) (see Supporting Information for copies of the ${ }^{1} \mathrm{H}$ NMR spectra for amides 10a$\mathbf{1}, \mathbf{4}$, and 10n-r, isolated by filtration).

The results obtained with the test set of 18 different $\mathrm{N}$ trimethylsilylated heteroaryl/arylamines in acetonitrile demonstrate the simplicity of the protocol and suggests its general applicability to a wide range of amines expressing weak nucleophilic character. Further, as extractive workup can be avoided and minimum waste is produced. This 'green' chemistry method ${ }^{16}$ can be readily adapted to rapid parallel synthesis of libraries of di(hetero)arylamide-based compounds.

All chemicals were purchased from Sigma Aldrich or Oakwood Chemicals and were used without purification, unless otherwise mentioned. All solvents were dried and kept under $\mathrm{N}_{2} .{ }^{1} \mathrm{H}$ and ${ }^{13} \mathrm{C}$ NMR spectra were recorded at 400 and $100 \mathrm{MHz}$, respectively, on a Bruker AC 400 Ultrashield 10 spectrometer. Chemical shifts are expressed in ppm ( $\delta$ scale). Standard abbreviations are used to report peak multiplicities. Coupling constants are reported in hertz $(\mathrm{Hz})$. High-resolution mass spectra was recorded on a Thermo Scientific Q Exactive Orbitrap High Resolution Mass Spectrometer. IR spectra $\left(\mathrm{cm}^{-1}\right)$ were recorded on a Agilent Technologies (Cary 600 series) FT-IR spectrometer, using a PIKE MIRacle ATR accessory for sampling. Melting points were obtained using a Mel-Temp II apparatus. 
4-(2-Methoxyethoxy)-1-methyl-6-oxo-1,6-dihydropyridine-3-carbonyl Fluoride ( 7 )

A mixture of $\mathbf{6}^{1 \mathrm{a}, \mathrm{b}}(1.0 \mathrm{~g}, 4.40 \mathrm{mmol})$, TFFH $(1.18 \mathrm{~g}, 4.40 \mathrm{mmol})$, and CsF $(1.57 \mathrm{~g}, 10.3 \mathrm{mmol})$ in $\mathrm{MeCN}(22 \mathrm{~mL})$ was stirred for $12 \mathrm{~h}$ at r.t. The mixture was then concentrated under reduced pressure and washed with hexanes to remove the tetramethylurea formed. The product mixture was then dissolved in $\mathrm{CH}_{2} \mathrm{Cl}_{2}$ and filtered to remove the excess CsF. The filtrate was concentrated and dried under high vacuum for 2 days to afford 7 as a beige solid $(0.91 \mathrm{~g}, 90 \%)$.

IR (ATR): 1814, 1778, 1678, 1617, $1530 \mathrm{~cm}^{-1}$.

${ }^{1} \mathrm{H} \mathrm{NMR}\left(400 \mathrm{MHz}, \mathrm{CDCl}_{3}\right): \delta=8.20(\mathrm{~s}, 1 \mathrm{H}), 5.91(\mathrm{~s}, 1 \mathrm{H}), 4.13(\mathrm{t}, J=4.3$ $\mathrm{Hz}, 2 \mathrm{H}), 3.81$ (t, J = 4.3 Hz, $2 \mathrm{H}), 3.57(\mathrm{~s}, 3 \mathrm{H}), 3.46(\mathrm{~s}, 3 \mathrm{H})$.

${ }^{13} \mathrm{C}$ NMR $\left(100 \mathrm{MHz}, \mathrm{CDCl}_{3}\right): \delta=165.6,163.5,154.1,150.8,148.1,97.3$, 70.1, 69.0, 59.6, 37.8.

${ }^{19} \mathrm{~F}$ NMR $\left(400 \mathrm{MHz}, \mathrm{CDCl}_{3}\right): \delta=25.43$.

\section{N-Trimethylsilylated Heteroaryl/arylamines TMS-9a-r; General Procedure}

The starting amines were dried under high vacuum for $12 \mathrm{~h}$. Then, a solution/mixture of the requisite amine $(0.5 \mathrm{mmol})$ in $\mathrm{Me}_{3} \mathrm{SiCN}(0.5$ $\mathrm{mL}$ ) was heated at $70{ }^{\circ} \mathrm{C}$ for the required time (Table 1 ). Without removing the hot oil bath, the resulting solution was flushed with $\mathrm{N}_{2}$ for $15 \mathrm{~min}$ and then concentrated under high vacuum The percent conversion of each amine to its $\mathrm{N}$-silylated derivative was determined by ${ }^{1} \mathrm{H}$ NMR analysis in $\mathrm{CDCl}_{3}$ (passed through a basic alumina column prior to use).

\section{$N$-[(6-Chloropyridin-3-yl)methyl]-1,1,1-trimethylsilanamine (TMS-9a)}

Heated for $7 \mathrm{~min}$; yellow oil; $91 \%$ conversion.

${ }^{1} \mathrm{H}$ NMR $\left(400 \mathrm{MHz}, \mathrm{CDCl}_{3}\right): \delta=8.22(\mathrm{~s}, 1 \mathrm{H}), 7.54(\mathrm{~d}, J=8.1 \mathrm{~Hz}, 1 \mathrm{H})$, $7.18(\mathrm{~d}, J=8.1 \mathrm{~Hz}, 1 \mathrm{H}), 3.85(\mathrm{~d}, J=8.2 \mathrm{~Hz}, 2 \mathrm{H}), 0.00(\mathrm{~s}, 9 \mathrm{H})$.

\section{5-Methyl- $\mathrm{N}$-(trimethylsilyl)isoxazol-3-amine (TMS-9b) $)^{12}$}

Heated for $30 \mathrm{~min}$; yellow solid; $100 \%$ conversion.

${ }^{1} \mathrm{H} \mathrm{NMR}\left(400 \mathrm{MHz}, \mathrm{CDCl}_{3}\right): \delta=5.46(\mathrm{~s}, 1 \mathrm{H}), 3.75(\mathrm{~s}, 1 \mathrm{H}), 2.24(\mathrm{~s}, 3 \mathrm{H})$, $0.23(\mathrm{~s}, 9 \mathrm{H})$.

\section{$\mathrm{N}$-(Trimethylsilyl)isoquinolin-4-amine (TMS-9c)}

Heated for $30 \mathrm{~min}$; yellow solid; $100 \%$ conversion.

${ }^{1} \mathrm{H} \mathrm{NMR}\left(400 \mathrm{MHz}, \mathrm{CDCl}_{3}\right.$ ): $\delta=8.62(\mathrm{~s}, 1 \mathrm{H}), 8.03(\mathrm{~s}, 1 \mathrm{H}), 7.79(\mathrm{~d}, J=$ $7.8 \mathrm{~Hz}, 1 \mathrm{H}), 7.73(\mathrm{~d}, J=8.4 \mathrm{~Hz}, 1 \mathrm{H}), 7.55(\mathrm{t}, J=7.7 \mathrm{~Hz}, 1 \mathrm{H}), 7.46(\mathrm{~d}, J=$ $7.7 \mathrm{~Hz}, 1 \mathrm{H}), 3.88(\mathrm{~s}, 1 \mathrm{H}), 0.30(\mathrm{~s}, 9 \mathrm{H})$.

\section{4-Methyl- $\mathbf{N}$-(trimethylsilyl)thiazol-2-amine (TMS-9d)}

Heated for $15 \mathrm{~min}$; yellow oil; $100 \%$ conversion.

${ }^{1} \mathrm{H} \mathrm{NMR}\left(400 \mathrm{MHz}, \mathrm{CDCl}_{3}\right): \delta=5.95(\mathrm{~s}, 1 \mathrm{H}), 4.84(\mathrm{~s}, 1 \mathrm{H}), 2.14(\mathrm{~s}, 3 \mathrm{H})$, $0.23(\mathrm{~s}, 9 \mathrm{H})$.

\section{3-Methyl- $\mathrm{N}$-(trimethylsilyl)isoxazol-5-amine (TMS-9e)}

Heated for $30 \mathrm{~min}$; orange solid; $100 \%$ conversion.

${ }^{1} \mathrm{H} \mathrm{NMR}\left(400 \mathrm{MHz}, \mathrm{CDCl}_{3}\right): \delta=4.78(\mathrm{~s}, 1 \mathrm{H}), 4.30(\mathrm{~s}, 1 \mathrm{H}), 2.08(\mathrm{~s}, 3 \mathrm{H})$, $0.21(\mathrm{~s}, 9 \mathrm{H})$.

\section{$\boldsymbol{N}$-(Trimethylsilyl)thiazol-2-amine (TMS-9f) ${ }^{13 a-d}$}

Heated for $30 \mathrm{~min}$; orange oil; $100 \%$ conversion.
${ }^{1} \mathrm{H}$ NMR (400 MHz, $\left.\mathrm{CDCl}_{3}\right): \delta=7.02(\mathrm{~s}, 1 \mathrm{H}), 6.40(\mathrm{~s}, 1 \mathrm{H}), 5.20(\mathrm{~s}, 1 \mathrm{H})$, $0.25(\mathrm{~s}, 9 \mathrm{H})$.

\section{Methyl 4-[(Trimethylsilyl)amino]benzoate (TMS-9g) ${ }^{14}$}

Heated for $180 \mathrm{~min}$; yellow oil; $100 \%$ conversion.

${ }^{1} \mathrm{H}$ NMR $\left(400 \mathrm{MHz}, \mathrm{CDCl}_{3}\right): \delta=7.68(\mathrm{~d}, J=7.8 \mathrm{~Hz}, 2 \mathrm{H}), 6.48(\mathrm{~d}, J=7.9$ $\mathrm{Hz}, 2 \mathrm{H}), 3.69$ (s, $4 \mathrm{H}), 0.14$ (s, $9 \mathrm{H})$.

\section{$\mathbf{N}$-(Trimethylsilyl)pyrazin-2-amine (TMS-9h) $)^{13 a}$}

Heated for 90 min; yellow oil; $100 \%$ conversion.

${ }^{1} \mathrm{H} \mathrm{NMR}\left(400 \mathrm{MHz}, \mathrm{CDCl}_{3}\right): \delta=7.83(\mathrm{t}, J=1.2 \mathrm{~Hz}, 3 \mathrm{H}), 4.23(\mathrm{~s}, 1 \mathrm{H})$, $0.24(\mathrm{~s}, 9 \mathrm{H})$.

\section{6-Chloro- $\mathbf{N}$-(trimethylsilyl)pyridin-3-amine (TMS-9i)}

Heated for $120 \mathrm{~min}$; purple solid; $100 \%$ conversion.

${ }^{1} \mathrm{H} \mathrm{NMR}\left(400 \mathrm{MHz}, \mathrm{CDCl}_{3}\right): \delta=7.73(\mathrm{~s}, 1 \mathrm{H}), 6.97(\mathrm{~d}, J=8.7 \mathrm{~Hz}, 1 \mathrm{H})$, $6.85(\mathrm{~d}, J=8.7 \mathrm{~Hz}, 1 \mathrm{H}), 3.44(\mathrm{~s}, 1 \mathrm{H}), 0.20(\mathrm{~s}, 9 \mathrm{H})$.

\section{$\mathbf{N}$-(Trimethylsilyl)benzo[d]thiazol-2-amine (TMS-9j)}

Heated for $30 \mathrm{~min}$; yellow oil; $100 \%$ conversion.

${ }^{1} \mathrm{H} \mathrm{NMR} \mathrm{(400} \mathrm{MHz,} \mathrm{CDCl}_{3}$ ): $\delta$ = 7.41 (s, $2 \mathrm{H}$ ), 7.13 (s, $\left.1 \mathrm{H}\right), 6.93$ (s, $1 \mathrm{H}$ ), $4.91(\mathrm{~s}, 1 \mathrm{H}), 0.21(\mathrm{~s}, 9 \mathrm{H})$.

\section{3-[(Trimethylsilyl)amino]benzonitrile (TMS-9k)}

Heated for $180 \mathrm{~min}$; yellow oil; $96 \%$ conversion.

${ }^{1} \mathrm{H} \mathrm{NMR}\left(400 \mathrm{MHz}, \mathrm{CDCl}_{3}\right): \delta=7.03(\mathrm{t}, J=7.8 \mathrm{~Hz}, 1 \mathrm{H}), 6.81(\mathrm{~d}, J=7.5$ $\mathrm{Hz}, 1 \mathrm{H}), 6.69$ (m, $2 \mathrm{H}), 3.51$ (s, $1 \mathrm{H}), 0.13(\mathrm{~s}, 9 \mathrm{H})$.

$\boldsymbol{N}$-(Trimethylsilyl)-1,3,4-thiadiazol-2-amine (TMS-91) ${ }^{15}$

Heated for $30 \mathrm{~min}$; yellow solid; $100 \%$ conversion.

$\left.{ }^{1} \mathrm{H} \mathrm{NMR} \mathrm{(400} \mathrm{MHz,} \mathrm{CDCl}_{3}\right): \delta=8.35(\mathrm{~s}, 1 \mathrm{H}), 6.01(\mathrm{~s}, 1 \mathrm{H}), 0.32(\mathrm{~s}, 9 \mathrm{H})$.

5-Nitro- $\mathbf{N}$-(trimethylsilyl)benzo[d]isothiazol-3-amine (TMS-9m) ${ }^{1 \mathrm{~b}}$ Heated for $30 \mathrm{~min}$; red/orange solid; $100 \%$ conversion.

${ }^{1} \mathrm{H}$ NMR (400 MHz, $\left.\mathrm{CDCl}_{3}\right): \delta=8.70(\mathrm{~s}, 1 \mathrm{H}), 7.99(\mathrm{~s}, 1 \mathrm{H}), 7.40(\mathrm{~s}, 1 \mathrm{H})$, $5.96(\mathrm{~s}, 1 \mathrm{H}), 0.43(\mathrm{~s}, 9 \mathrm{H})$.

4-Chloro- $\boldsymbol{N}$-(trimethylsilyl)benzo[d]thiazol-2-amine (TMS-9n)

Heated for $30 \mathrm{~min}$; yellow oil; $100 \%$ conversion.

${ }^{1} \mathrm{H} \mathrm{NMR}\left(400 \mathrm{MHz}, \mathrm{CDCl}_{3}\right): \delta=7.37(\mathrm{~d}, J=7.7 \mathrm{~Hz}, 1 \mathrm{H}), 7.23$ (d, $J=7.8$ $\mathrm{Hz}, 1 \mathrm{H}), 6.92(\mathrm{t}, J=7.7 \mathrm{~Hz}, 1 \mathrm{H}), 5.22(\mathrm{~s}, 1 \mathrm{H}), 0.30(\mathrm{~s}, 9 \mathrm{H})$.

4-[(Trimethylsilyl)amino]benzonitrile (TMS-9o $)^{13 a}$

Heated for $180 \mathrm{~min}$; yellow oil; $100 \%$ conversion.

${ }^{1} \mathrm{H} \mathrm{NMR}\left(400 \mathrm{MHz}, \mathrm{CDCl}_{3}\right): \delta=7.23(\mathrm{~d}, J=8.0 \mathrm{~Hz}, 2 \mathrm{H}), 6.49(\mathrm{~d}, J=8.0$ $\mathrm{Hz}, 2 \mathrm{H}), 3.79$ (s, $1 \mathrm{H}), 0.14$ (s, $9 \mathrm{H})$.

\section{5-(2-Chlorophenyl)- $N$-(trimethylsilyl)-1,3,4-oxadiazol-2-amine (TMS-9p)}

Heated for $60 \mathrm{~min}$; white solid; $100 \%$ conversion.

${ }^{1} \mathrm{H}$ NMR $\left(400 \mathrm{MHz}, \mathrm{CDCl}_{3}\right): \delta=7.85(\mathrm{~d}, J=7.3 \mathrm{~Hz}, 1 \mathrm{H}), 7.43(\mathrm{~d}, J=7.5$ $\mathrm{Hz}, 1 \mathrm{H}), 7.30$ (m, $2 \mathrm{H}), 5.45$ (s, $1 \mathrm{H}), 0.32$ (s, $9 \mathrm{H})$.

2-Chloro- $\mathbf{N}$-(trimethylsilyl)pyridin-4-amine (TMS-9q) Heated for $30 \mathrm{~min}$; orange oil; $100 \%$ conversion. 
${ }^{1} \mathrm{H}$ NMR $\left(400 \mathrm{MHz}, \mathrm{CDCl}_{3}\right): \delta=7.87(\mathrm{~d}, J=5.7 \mathrm{~Hz}, 1 \mathrm{H}), 6.47(\mathrm{~s}, 1 \mathrm{H})$, $6.38(\mathrm{~d}, J=5.6 \mathrm{~Hz}, 1 \mathrm{H}), 4.09$ (s, $1 \mathrm{H}), 0.24(\mathrm{~s}, 9 \mathrm{H})$.

\section{5-[(Trimethylsilyl)amino]picolinonitrile (TMS-9r)}

Heated for $6 \mathrm{~h}$; orange solid; $85 \%$ conversion.

${ }^{1} \mathrm{H} \mathrm{NMR}\left(400 \mathrm{MHz}, \mathrm{CDCl}_{3}\right): \delta=8.01(\mathrm{~s}, 1 \mathrm{H}), 7.37(\mathrm{~d}, J=8.6 \mathrm{~Hz}, 1 \mathrm{H})$, $6.88(\mathrm{~d}, J=8.6 \mathrm{~Hz}, 1 \mathrm{H}), 3.99$ (s, $1 \mathrm{H}), 0.26(\mathrm{~s}, 9 \mathrm{H})$.

\section{Di(hetero)arylamides 10a-r; General Procedure}

Acid fluoride 7 (200 mg, $0.89 \mathrm{mmol}$ ) in $\mathrm{MeCN}(5 \mathrm{~mL})$ was added in one portion to the silylated amine $\mathbf{9 a}-\mathbf{r}(0.50 \mathrm{mmol})$ at r.t. This was quickly followed by addition of $1 \mathrm{M} \mathrm{TBAF}$ in THF ( $10 \mu \mathrm{L}, 0.01 \mathrm{mmol})$. The reaction mixture/solution was stirred at $50{ }^{\circ} \mathrm{C}$ for $12 \mathrm{~h}$. The precipitated product was collected with suction filtration and washed with $\mathrm{MeCN}$ or the specified solvent (Table 1).

$N$-[(6-Chloropyridin-3-yl)methyl]-4-(2-methoxyethoxy)-1-methyl-6-oxo-1,6-dihydropyridine-3-carboxamide (10a)

From TMS-amine 9a. The precipitated product was washed with $\mathrm{MeCN} / \mathrm{Et}_{2} \mathrm{O}$ (1:1) to afford 10a as a white solid; yield: $274 \mathrm{mg}$ (78\%); mp $187-188^{\circ} \mathrm{C}$.

IR (ATR): 3393, 1675, 1632, $1528 \mathrm{~cm}^{-1}$.

${ }^{1} \mathrm{H}$ NMR (400 MHz, DMSO- $d_{6}$ ): $\delta=8.37(\mathrm{~d}, J=2.4 \mathrm{~Hz}, 1 \mathrm{H}), 8.28(\mathrm{~m}, 2$ $\mathrm{H}), 7.78(\mathrm{dd}, J=2.5,8.2 \mathrm{~Hz}, 1 \mathrm{H}), 7.50(\mathrm{~d}, J=8.2 \mathrm{~Hz}, 1 \mathrm{H}), 5.92(\mathrm{~s}, 1 \mathrm{H})$, 4.49 (d, J = 5.9 Hz, 2 H), 4.19 (m, 2 H), 3.67 (m, 2 H), 3.42 (s, 3 H), 3.19 (s, $3 \mathrm{H})$.

${ }^{13} \mathrm{C}$ NMR $\left(100 \mathrm{MHz}\right.$, DMSO- $\left.d_{6}\right): \delta=163.7,162.7,162.5,148.9,148.8$, $144.5,138.9,134.5,124.0,105.7,96.3,69.3,68.0,58.1,36.3$.

HRMS (HESI): $m / z$ [M - H] $]^{-}$calcd for $\mathrm{C}_{16} \mathrm{H}_{17} \mathrm{ClN}_{3} \mathrm{O}_{4}$ : 350.0913; found: 350.0916 .

\section{4-(2-Methoxyethoxy)-1-methyl- $\mathrm{N}$-(5-methylisoxazol-3-yl)-6-oxo-} 1,6-dihydropyridine-3-carboxamide (10b)

From TMS-amine $\mathbf{9 b}$. The precipitated product was washed with $\mathrm{MeCN}$ to afford $\mathbf{1 0 b}$ as a pale yellow solid; yield: $21 \mathrm{mg}$ (7\%); mp 179$181^{\circ} \mathrm{C}$.

IR (ATR): 3337, 1685, 1660, 1612, $1548 \mathrm{~cm}^{-1}$.

${ }^{1} \mathrm{H} \mathrm{NMR}\left(400 \mathrm{MHz}, \mathrm{CDCl}_{3}\right): \delta=9.98(\mathrm{~s}, 1 \mathrm{H}), 8.36(\mathrm{~s}, 1 \mathrm{H}), 6.71(\mathrm{~s}, 1 \mathrm{H})$, $5.94(\mathrm{~s}, 1 \mathrm{H}), 4.24(\mathrm{t}, J=3.8 \mathrm{~Hz}, 2 \mathrm{H}), 3.85(\mathrm{t}, J=3.7 \mathrm{~Hz}, 2 \mathrm{H}), 3.58(\mathrm{~s}, 3$ $\mathrm{H}), 3.54(\mathrm{~s}, 3 \mathrm{H}), 2.41(\mathrm{~s}, 3 \mathrm{H})$.

${ }^{13} \mathrm{C}$ NMR $\left(100 \mathrm{MHz}, \mathrm{CDCl}_{3}\right): \delta=169.8,163.9,163.8,160.8,158.2$, $145.9,105.6,97.3,97.1,69.6,68.7,59.6,37.7,12.8$.

HRMS (HESI): $m / z$ [M - H] $]^{+}$calcd for $\mathrm{C}_{14} \mathrm{H}_{16} \mathrm{~N}_{3} \mathrm{O}_{5}$ : 306.1095; found: 306.1102 .

\section{$N$-(Isoquinolin-4-yl)-4-(2-methoxyethoxy)-1-methyl-6-oxo-1,6- dihydropyridine-3-carboxamide (10c)}

From TMS-amine $\mathbf{9 c}$. The precipitated product was washed with $\mathrm{Et}_{2} \mathrm{O}$ to afford 10c as a white solid; yield: $95 \mathrm{mg}(27 \%) ; \mathrm{mp} 213-214^{\circ} \mathrm{C}$.

IR (ATR): 3286, 1664, 1627, $1543 \mathrm{~cm}^{-1}$.

${ }^{1} \mathrm{H}$ NMR $\left(400 \mathrm{MHz}\right.$, DMSO- $\left.d_{6}\right): \delta=9.88(\mathrm{~s}, 1 \mathrm{H}), 9.20(\mathrm{~s}, 1 \mathrm{H}), 8.85(\mathrm{~s}, 1$ $\mathrm{H}), 8.48(\mathrm{~s}, 1 \mathrm{H}), 8.20(\mathrm{~d}, J=8.5 \mathrm{~Hz}, 1 \mathrm{H}), 8.06(\mathrm{~d}, J=8.5 \mathrm{~Hz}, 1 \mathrm{H}), 7.87$ $(\mathrm{d}, J=15.2 \mathrm{~Hz}, 1 \mathrm{H}), 7.75(\mathrm{~d}, J=15.2 \mathrm{~Hz}, 1 \mathrm{H}), 6.07(\mathrm{~s}, 1 \mathrm{H}), 4.36(\mathrm{~m}, 2$ H), $3.80(\mathrm{~m}, 2 \mathrm{H}), 3.50(\mathrm{~s}, 3 \mathrm{H}), 3.19(\mathrm{~s}, 3 \mathrm{H})$.

${ }^{13} \mathrm{C}$ NMR $\left(100 \mathrm{MHz}\right.$, DMSO- $\left.d_{6}\right): \delta=163.6,162.8,161.8,149.5,145.3$, 138.4, 130.7, 130.0, 128.4, 128.3, 127.9, 127.7, 121.3, 105.8, 96.5, 69.4, $68.5,58.2,36.4$.
HRMS (HESI): $m / z[M+H]^{+}$calcd for $\mathrm{C}_{19} \mathrm{H}_{20} \mathrm{~N}_{3} \mathrm{O}_{4}: 354.1448$; found: 354.1449 .

4-(2-Methoxyethoxy)-1-methyl- $N$-(4-methylthiazol-2-yl)-6-oxo1,6-dihydropyridine-3-carboxamide (10d)

From TMS-amine 9d. The precipitated product was washed with $\mathrm{Et}_{2} \mathrm{O}$ to afford 10d as a yellow solid; yield: $110 \mathrm{mg}(34 \%)$; mp $189-190{ }^{\circ} \mathrm{C}$. IR (ATR): 3308, 1680, 1635, $1530 \mathrm{~cm}^{-1}$.

${ }^{1} \mathrm{H}$ NMR $\left(400 \mathrm{MHz}\right.$, DMSO- $\left.d_{6}\right): \delta=11.07(\mathrm{~s}, 1 \mathrm{H}), 8.48(\mathrm{~s}, 1 \mathrm{H}), 6.81(\mathrm{~s}$, $1 \mathrm{H}), 6.00(\mathrm{~s}, 1 \mathrm{H}), 4.27(\mathrm{~m}, 2 \mathrm{H}), 3.75(\mathrm{~m}, 2 \mathrm{H}), 3.47(\mathrm{~s}, 3 \mathrm{H}), 3.40(\mathrm{~s}, 3$ $\mathrm{H}), 2.27(\mathrm{~s}, 3 \mathrm{H})$.

${ }^{13} \mathrm{C}$ NMR $\left(100 \mathrm{MHz}\right.$, DMSO- $\left.d_{6}\right): \delta=163.3,162.7,160.3,156.5,147.0$, $145.8,108.4,104.0,96.4,69.4,68.5,58.5,36.5,16.9$.

HRMS (HESI): $m / z$ [M $+\mathrm{H}]^{+}$calcd for $\mathrm{C}_{14} \mathrm{H}_{18} \mathrm{~N}_{3} \mathrm{O}_{4} \mathrm{~S}$ : 324.1013; found: 324.1015 .

4-(2-Methoxyethoxy)-1-methyl- $N$-(3-methylisoxazol-5-yl)-6-oxo1,6-dihydropyridine-3-carboxamide (10e)

From TMS-amine 9e. The precipitated product was washed with $\mathrm{Et}_{2} \mathrm{O}$ to afford 10e as a white solid; yield: $163 \mathrm{mg}(53 \%) ; \mathrm{mp} 211-213^{\circ} \mathrm{C}$.

IR (ATR): 3306, 1694, 1656, 1610, $1528 \mathrm{~cm}^{-1}$.

${ }^{1} \mathrm{H}$ NMR $\left(400 \mathrm{MHz}\right.$, DMSO- $\left.d_{6}\right): \delta=10.72(\mathrm{~s}, 1 \mathrm{H}), 8.42(\mathrm{~s}, 1 \mathrm{H}), 6.24(\mathrm{~s}$, $1 \mathrm{H}), 5.99(\mathrm{~s}, 1 \mathrm{H}), 4.25$ (m, $2 \mathrm{H}), 3.74(\mathrm{~m}, 2 \mathrm{H}), 3.46$ (s, $3 \mathrm{H}), 3.36$ (s, 3 $\mathrm{H}), 2.21(\mathrm{~s}, 3 \mathrm{H})$.

${ }^{13} \mathrm{C}$ NMR $\left(100 \mathrm{MHz}\right.$, DMSO- $\left.d_{6}\right): \delta=162.7,160.8,160.4,159.1,145.7$, 104.6, 96.4, 89.3, 69.4, 68.3, 58.3, 36.5, 11.4.

HRMS (HESI): $m / z$ [M - H] $]^{-}$calcd for $\mathrm{C}_{14} \mathrm{H}_{16} \mathrm{~N}_{3} \mathrm{O}_{5}$ : 306.1095; found: 306.1102 .

4-(2-Methoxyethoxy)-1-methyl-6-oxo- $N$-(thiazol-2-yl)-1,6-dihydropyridine-3-carboxamide (10f)

From TMS-amine $\mathbf{9 f}$. The precipitated product was washed with $\mathrm{Et}_{2} \mathrm{O}$ to afford 10f as a pale yellow solid; yield: $170 \mathrm{mg}$ (55\%); mp 185-186 ${ }^{\circ} \mathrm{C}$.

IR (ATR): 3311, 1678, 1632, 1612, $1530 \mathrm{~cm}^{-1}$.

${ }^{1} \mathrm{H}$ NMR (400 MHz, DMSO- $d_{6}$ ): $\delta=11.14(\mathrm{~s}, 1 \mathrm{H}), 8.50(\mathrm{~s}, 1 \mathrm{H}), 7.50(\mathrm{~d}$, $J=3.5 \mathrm{~Hz}, 1 \mathrm{H}), 7.27(\mathrm{~d}, J=3.5 \mathrm{~Hz}, 1 \mathrm{H}), 6.01(\mathrm{~s}, 1 \mathrm{H}), 4.28(\mathrm{~m}, 2 \mathrm{H})$, $3.76(\mathrm{~m}, 2 \mathrm{H}), 3.47$ (s, $3 \mathrm{H}), 3.38(\mathrm{~s}, 3 \mathrm{H})$.

${ }^{13} \mathrm{C}$ NMR $\left(100 \mathrm{MHz}\right.$, DMSO- $\left.d_{6}\right): \delta=163.3,162.7,160.4,157.3,145.8$, 138.0, 114.2, 104.0, 96.4, 69.4, 68.5, 58.5, 36.5.

HRMS (HESI): $m / z$ [M - H] $]^{-}$calcd for $\mathrm{C}_{13} \mathrm{H}_{14} \mathrm{~N}_{3} \mathrm{O}_{4} \mathrm{~S}$ : 308.0711; found: 308.0696 .

Methyl 4-[4-(2-Methoxyethoxy)-1-methyl-6-oxo-1,6-dihydropyridine-3-carboxamido]benzoate $(\mathbf{1 0 g})$

From TMS-amine 9g. The precipitated product was washed with MeCN to afford $\mathbf{1 0 g}$ as a white solid; yield: $321 \mathrm{mg}$ (89\%); $\mathrm{mp} 213-$ $215^{\circ} \mathrm{C}$.

IR (ATR): 3348, 1688, 1656, 1592, $1527 \mathrm{~cm}^{-1}$.

${ }^{1} \mathrm{H}$ NMR $\left(400 \mathrm{MHz}, \mathrm{CDCl}_{3}\right): \delta=9.76(\mathrm{~s}, 1 \mathrm{H}), 8.41(\mathrm{~s}, 1 \mathrm{H}), 8.03(\mathrm{~d}, J=$ $8.6 \mathrm{~Hz}, 2 \mathrm{H}), 7.73(\mathrm{~d}, J=8.6 \mathrm{~Hz}, 2 \mathrm{H}), 6.04(\mathrm{~s}, 1 \mathrm{H}), 4.28(\mathrm{t}, J=3.9 \mathrm{~Hz}, 2$ $\mathrm{H}), 3.90(\mathrm{~m}, 5 \mathrm{H}), 3.61(\mathrm{~s}, 3 \mathrm{H}), 3.53(\mathrm{~s}, 3 \mathrm{H})$.

${ }^{13} \mathrm{C}$ NMR $\left(100 \mathrm{MHz}, \mathrm{CDCl}_{3}\right): \delta=166.8,163.9,163.7,161.1,145.7$, 142.7, 130.9, 125.6, 119.5, 106.3, 97.3, 69.8, 68.0, 59.1, 52.2, 37.7.

HRMS (HESI): $m / z$ [M - H] calcd for $\mathrm{C}_{18} \mathrm{H}_{19} \mathrm{~N}_{2} \mathrm{O}_{6}$ : 359.1249; found: 359.1251 . 
4-(2-Methoxyethoxy)-1-methyl-6-oxo- $N$-(pyrazin-2-yl)-1,6-dihydropyridine-3-carboxamide (10h)

From TMS-amine $\mathbf{9 h}$. The precipitated product was washed with $\mathrm{Et}_{2} \mathrm{O}$ to afford 10h as a white solid; yield: $128 \mathrm{mg}(42 \%)$; mp 191-193 ${ }^{\circ} \mathrm{C}$.

IR (ATR): 3331, 1693, 1645, $1528 \mathrm{~cm}^{-1}$.

${ }^{1} \mathrm{H}$ NMR $\left(400 \mathrm{MHz}\right.$, DMSO- $\left.d_{6}\right): \delta=10.20(\mathrm{~s}, 1 \mathrm{H}), 9.44(\mathrm{~d}, J=1.5 \mathrm{~Hz}, 1$ H), $8.53(\mathrm{~s}, 1 \mathrm{H}), 8.43(\mathrm{~m}, 2 \mathrm{H}), 6.01(\mathrm{~s}, 1 \mathrm{H}), 4.29(\mathrm{~m}, 2 \mathrm{H}), 3.76(\mathrm{~m}, 2$ $\mathrm{H}), 3.49(\mathrm{~s}, 3 \mathrm{H}), 3.36(\mathrm{~s}, 3 \mathrm{H})$.

${ }^{13} \mathrm{C}$ NMR (100 MHz, DMSO- $\left.d_{6}\right): \delta=163.3,162.7,161.1,148.3,146.2$, 142.9, 140.2, 136.3, 104.5, 96.4, 69.5, 68.8, 58.4, 36.6.

HRMS (HESI): $m / z[\mathrm{M}+\mathrm{Na}]^{+}$calcd for $\mathrm{C}_{14} \mathrm{H}_{16} \mathrm{~N}_{4} \mathrm{O}_{4} \mathrm{Na}$ : 327.1064; found: 327.1062 .

\section{$N$-(6-Chloropyridin-3-yl)-4-(2-methoxyethoxy)-1-methyl-6-oxo- 1,6-dihydropyridine-3-carboxamide (10i)}

From TMS-amine 9i. The precipitated product was washed with MeCN to afford 10i as a white solid; yield: 209 mg (62\%); mp 195-196 ${ }^{\circ} \mathrm{C}$.

IR (ATR): 3329, 1694, 1649, 1596, $1529 \mathrm{~cm}^{-1}$.

${ }^{1} \mathrm{H}$ NMR (400 MHz, DMSO- $\left.d_{6}\right): \delta=9.96(\mathrm{~s}, 1 \mathrm{H}), 8.63(\mathrm{~d}, J=2.5 \mathrm{~Hz}, 1$ $\mathrm{H}), 8.41(\mathrm{~s}, 1 \mathrm{H}), 8.16(\mathrm{dd}, J=2.8,8.7 \mathrm{~Hz}, 1 \mathrm{H}), 7.54(\mathrm{~d}, J=8.8 \mathrm{~Hz}, 1 \mathrm{H})$, 6.00 (s, $1 \mathrm{H}), 4.27$ (m, $2 \mathrm{H}), 3.79$ (m, $2 \mathrm{H}), 3.47$ (s, $3 \mathrm{H}), 3.33$ (s, $3 \mathrm{H})$.

${ }^{13} \mathrm{C}$ NMR $\left(100 \mathrm{MHz}\right.$, DMSO- $\left.d_{6}\right): \delta=163.3,162.7,161.4,145.2,143.9$, $140.8,134.9,130.2,124.3,105.6,96.3,69.4,67.9,58.1,36.5$.

HRMS (HESI): $m / z$ [M - H] $]^{-}$calcd for $\mathrm{C}_{15} \mathrm{H}_{15} \mathrm{ClN}_{3} \mathrm{O}_{4}$ : 336.0757; found: 336.0765 .

\section{$N$-(Benzo[d]thiazol-2-yl)-4-(2-methoxyethoxy)-1-methyl-6-oxo- 1,6-dihydropyridine-3-carboxamide (10j)}

From TMS-amine 9j. The precipitated product was washed with $\mathrm{MeCN}$ to afford $\mathbf{1 0 j}$ as a white solid; yield: $255 \mathrm{mg}$ (71\%); mp 209-211 ${ }^{\circ} \mathrm{C}$.

IR (ATR): 3302, 1684, 1640, 1600, $1530 \mathrm{~cm}^{-1}$.

${ }^{1} \mathrm{H} \mathrm{NMR}\left(400 \mathrm{MHz}, \mathrm{CDCl}_{3}\right): \delta=11.01(\mathrm{~s}, 1 \mathrm{H}), 8.45(\mathrm{~s}, 1 \mathrm{H}), 7.81(\mathrm{dd}, J=$ 7.9, $19.0 \mathrm{~Hz}, 2 \mathrm{H}), 7.44(\mathrm{t}, J=7.5 \mathrm{~Hz}, 1 \mathrm{H}), 7.30(\mathrm{t}, J=7.5 \mathrm{~Hz}, 1 \mathrm{H}), 5.99$ (s, $1 \mathrm{H}), 4.34(\mathrm{~s}, 2 \mathrm{H}), 3.94(\mathrm{~s}, 2 \mathrm{H}), 3.64(\mathrm{~s}, 3 \mathrm{H}), 3.60(\mathrm{~s}, 3 \mathrm{H})$.

${ }^{13} \mathrm{C}$ NMR $\left(100 \mathrm{MHz}, \mathrm{CDCl}_{3}\right): \delta=163.7,161.1,157.8,148.4,146.3$, 132.4, 126.3, 124.1, 121.5, 121.1, 104.4, 97.5, 69.8, 68.9, 59.7, 37.8 .

HRMS (HESI): $m / z$ [M - H] $]^{-}$calcd for $\mathrm{C}_{17} \mathrm{H}_{16} \mathrm{~N}_{3} \mathrm{O}_{4} \mathrm{~S}$ : 358.0867; found: 358.0849 .

N-(3-Cyanophenyl)-4-(2-methoxyethoxy)-1-methyl-6-oxo-1,6-dihydropyridine-3-carboxamide (10k)

From TMS-amine 9k. The precipitated product was washed with $\mathrm{MeCN}$ and $\mathrm{MeOH}$ to afford 10k as a white solid; yield: $278 \mathrm{mg}(85 \%)$; $\mathrm{mp} 236-238^{\circ} \mathrm{C}$.

IR (ATR): 3356, 2227, 1690, 1649, 1591, $1555 \mathrm{~cm}^{-1}$.

${ }^{1} \mathrm{H}$ NMR $\left(400 \mathrm{MHz}\right.$, DMSO- $\left.d_{6}\right): \delta=9.98(\mathrm{~s}, 1 \mathrm{H}), 8.40(\mathrm{~s}, 1 \mathrm{H}), 8.09(\mathrm{~s}, 1$ H), $7.90(\mathrm{td}, J=1.9,7.6 \mathrm{~Hz}, 1 \mathrm{H}), 7.58(\mathrm{~m}, 2 \mathrm{H}), 5.99(\mathrm{~s}, 1 \mathrm{H}), 4.27(\mathrm{~m}, 2$ $\mathrm{H}), 3.80(\mathrm{~m}, 2 \mathrm{H}), 3.47(\mathrm{~s}, 3 \mathrm{H}), 3.35(\mathrm{~s}, 3 \mathrm{H})$.

${ }^{13} \mathrm{C}$ NMR $\left(100 \mathrm{MHz}\right.$, DMSO- $\left.d_{6}\right): \delta=163.3,162.7,161.3,145.2,139.4$, 130.4, 127.2, 124.1, 122.3, 118.6, 111.7, 105.7, 96.3, 69.4, 68.0, 58.0, 36.5.
HRMS (HESI): $m / z$ [M - H] $]^{-}$calcd for $\mathrm{C}_{17} \mathrm{H}_{16} \mathrm{~N}_{3} \mathrm{O}_{4}$ : 326.1146; found: 326.1152 .

4-(2-Methoxyethoxy)-1-methyl-6-oxo- $N$-(1,3,4-thiadiazol-2-yl)1,6-dihydropyridine-3-carboxamide (101)

From TMS-amine 91. The precipitated product was washed with MeCN to afford 101 as a white solid; yield: $270 \mathrm{mg}$ (87\%); mp 234-236 ${ }^{\circ} \mathrm{C}$.

IR (ATR): 3308, 1686, 1636, 1611, $1528 \mathrm{~cm}^{-1}$.

${ }^{1} \mathrm{H}$ NMR $\left(400 \mathrm{MHz}\right.$, DMSO- $\left.d_{6}\right): \delta=11.51(\mathrm{~s}, 1 \mathrm{H}), 9.20(\mathrm{~s}, 1 \mathrm{H}), 8.51(\mathrm{~s}$, $1 \mathrm{H}), 6.01$ (s, $1 \mathrm{H}), 4.28$ (m, $2 \mathrm{H}), 3.76(\mathrm{~m}, 2 \mathrm{H}), 3.47$ (s, $3 \mathrm{H}), 3.39$ (s, 3 $\mathrm{H})$.

${ }^{13} \mathrm{C}$ NMR $\left(100 \mathrm{MHz}\right.$, DMSO- $\left.d_{6}\right): \delta=163.4,162.7,160.9,158.2,149.2$, 146.0, 103.8, 96.4, 69.4, 68.5, 58.5, 36.5.

HRMS (HESI): $m / z$ [M - H] $]^{-}$calcd for $\mathrm{C}_{12} \mathrm{H}_{13} \mathrm{~N}_{4} \mathrm{O}_{4} \mathrm{~S}$ : 309.0663; found: 309.0647 .

4-(2-Methoxyethoxy)-1-methyl- $N$-(5-nitrobenzo[d]isothiazol-3yl)-6-oxo-1,6-dihydropyridine-3-carboxamide (4)

From TMS-amine $\mathbf{9 m}$. The precipitated product was washed with MeCN and acetone to afford 4 as a yellow solid; yield: $242 \mathrm{mg}(60 \%)$; mp $296-298^{\circ} \mathrm{C}$.

IR (ATR): 3297, 1692, 1638, 1610, $1511 \mathrm{~cm}^{-1}$.

${ }^{1} \mathrm{H} /{ }^{13} \mathrm{C}$ NMR: See references $1 \mathrm{a}, \mathrm{b}$.

HRMS (HESI): $m / z$ [M - H] $]^{-}$calcd for $\mathrm{C}_{17} \mathrm{H}_{15} \mathrm{~N}_{4} \mathrm{O}_{6} \mathrm{~S}$ : 403.0718; found: 403.0695.

$\mathrm{N}$-(4-Chlorobenzo[d]thiazol-2-yl)-4-(2-methoxyethoxy)-1-methyl-6-oxo-1,6-dihydropyridine-3-carboxamide (10n)

From TMS-amine 9n. The precipitated product was washed with $\mathrm{MeCN}$ to afford 10n as a white solid; yield: $362 \mathrm{mg}$ (92\%); mp 266$267^{\circ} \mathrm{C}$.

IR (ATR): 3288, 1684, 1638, 1589, $1527 \mathrm{~cm}^{-1}$.

${ }^{1} \mathrm{H}$ NMR (400 MHz, DMSO- $d_{6}$ ): $\delta=11.61(\mathrm{~s}, 1 \mathrm{H}), 8.55(\mathrm{~s}, 1 \mathrm{H}), 7.99$ $(\mathrm{dd}, J=1.0,7.9 \mathrm{~Hz}, 1 \mathrm{H}), 7.54(\mathrm{dd}, J=1.0,7.8 \mathrm{~Hz}, 1 \mathrm{H}), 7.32(\mathrm{t}, J=7.9 \mathrm{~Hz}$, $1 \mathrm{H}), 6.02(\mathrm{~s}, 1 \mathrm{H}), 4.29(\mathrm{~m}, 2 \mathrm{H}), 3.79(\mathrm{~m}, 2 \mathrm{H}), 3.48(\mathrm{~s}, 6 \mathrm{H})$.

${ }^{13} \mathrm{C}$ NMR $\left(100 \mathrm{MHz}\right.$, DMSO- $\left.d_{6}\right): \delta=163.4,162.7,161.7,158.4,146.2$, 145.4, 133.4, 126.3, 124.6, 124.5, 120.9, 103.8, 96.4, 69.5, 68.6, 58.8, 36.5 .

HRMS (HESI): $m / z$ [M - H] $]^{-}$calcd for $\mathrm{C}_{17} \mathrm{H}_{15} \mathrm{ClN}_{3} \mathrm{O}_{4} \mathrm{~S}: 392.0477$; found: 392.0461 .

$N$-(4-Cyanophenyl)-4-(2-methoxyethoxy)-1-methyl-6-oxo-1,6-dihydropyridine-3-carboxamide (100)

From TMS-amine 9o. The precipitated product was washed with $\mathrm{MeOH}$ to afford 100 as a white solid; yield: $265 \mathrm{mg}$ (81\%); $\mathrm{mp}$ 249$251^{\circ} \mathrm{C}$.

IR (ATR): 3364, 2219, 1687, 1645, 1589, $1527 \mathrm{~cm}^{-1}$.

${ }^{1} \mathrm{H}$ NMR $\left(400 \mathrm{MHz}\right.$, DMSO- $\left.d_{6}\right): \delta=10.06(\mathrm{~s}, 1 \mathrm{H}), 8.40(\mathrm{~s}, 1 \mathrm{H}), 7.83(\mathrm{~s}$, $4 \mathrm{H}), 5.99$ (s, $1 \mathrm{H}), 4.27$ (m, $2 \mathrm{H}), 3.78(\mathrm{~m}, 2 \mathrm{H}), 3.47$ (s, $3 \mathrm{H}), 3.33$ (s, 3 $\mathrm{H})$.

${ }^{13} \mathrm{C}$ NMR $\left(100 \mathrm{MHz}\right.$, DMSO- $\left.d_{6}\right): \delta=163.3,162.7,161.4,145.2,142.8$, 133.4, 119.6, 119.0, 105.9, 105.4, 96.3, 69.4, 68.0, 58.1, 36.4.

HRMS (HESI): $m / z$ [M - H] $]^{-}$calcd for $\mathrm{C}_{17} \mathrm{H}_{16} \mathrm{~N}_{3} \mathrm{O}_{4}$ : 326.1146; found: 326.1153. 
$N$-[5-(2-Chlorophenyl)-1,3,4-oxadiazol-2-yl]-4-(2-methoxyethoxy)-1-methyl-6-oxo-1,6-dihydropyridine-3-carboxamide (10p) From TMS-amine 9p. The precipitated product was washed with MeCN to afford 10p as a pale yellow solid; yield: $255 \mathrm{mg}(63 \%)$; $\mathrm{mp}$ $190-192{ }^{\circ} \mathrm{C}$.

IR (ATR): 3289, 1698, 1667, 1594, $1528 \mathrm{~cm}^{-1}$.

${ }^{1} \mathrm{H}$ NMR (400 MHz, DMSO- $d_{6}$ ): $\delta=11.09(\mathrm{~s}, 1 \mathrm{H}), 8.41(\mathrm{~s}, 1 \mathrm{H}), 7.93$ (dd, $J=1.7,7.7 \mathrm{~Hz}, 1 \mathrm{H}$ ), 7.64 (dddd, $J=1.2,7.8,15.0,27 \mathrm{~Hz}, 3 \mathrm{H}$ ), 5.98 (s, $1 \mathrm{H}), 4.22(\mathrm{~m}, 2 \mathrm{H}), 3.71(\mathrm{~m}, 2 \mathrm{H}), 3.46(\mathrm{~s}, 3 \mathrm{H}), 3.31(\mathrm{~s}, 3 \mathrm{H})$.

${ }^{13} \mathrm{C}$ NMR $\left(100 \mathrm{MHz}\right.$, DMSO- $\left.d_{6}\right): \delta=163.3,162.7,160.4,158.9,157.6$, 145.6, 133.1, 131.6, 131.1, 131.0, 127.9, 122.5, 105.1, 96.3, 69.4, 68.4, $58.3,36.5$.

HRMS (HESI): $m / z$ [M - H] $]^{-}$calcd for $\mathrm{C}_{18} \mathrm{H}_{16} \mathrm{ClN}_{4} \mathrm{O}_{5}$ : 403.0815; found: 403.0823.

\section{$\mathrm{N}$-(2-Chloropyridin-4-yl)-4-(2-methoxyethoxy)-1-methyl-6-oxo- 1,6-dihydropyridine-3-carboxamide (10q)}

From TMS-amine 9q. The precipitated product was washed with $\mathrm{MeCN}$ to afford 10q as a white solid; yield: $148 \mathrm{mg}$ (44\%); $\mathrm{mp} 228-$ $229^{\circ} \mathrm{C}$.

IR (ATR): 3332, 1700, 1655, 1580, $1509 \mathrm{~cm}^{-1}$.

${ }^{1} \mathrm{H}$ NMR (400 MHz, DMSO- $\left.d_{6}\right): \delta=10.16(\mathrm{~s}, 1 \mathrm{H}), 8.41(\mathrm{~s}, 1 \mathrm{H}), 8.32$ (d, $J=5.6 \mathrm{~Hz}, 1 \mathrm{H}), 7.75(\mathrm{~d}, J=1.5 \mathrm{~Hz}, 1 \mathrm{H}), 7.58(\mathrm{dd}, J=1.7,5.6 \mathrm{~Hz}, 1 \mathrm{H})$, $6.00(\mathrm{~s}, 1 \mathrm{H}), 4.26(\mathrm{~m}, 2 \mathrm{H}), 3.78(\mathrm{~m}, 2 \mathrm{H}), 3.47(\mathrm{~s}, 3 \mathrm{H}), 3.34(\mathrm{~s}, 3 \mathrm{H})$.

${ }^{13} \mathrm{C}$ NMR $\left(100 \mathrm{MHz}\right.$, DMSO- $\left.d_{6}\right): \delta=163.7,163.1,162.5,151.5,151.0$, 148.2, 146.0, 113.5, 113.3, 106.0, 96.7, 69.9, 68.5, 58.5, 37.0.

HRMS (HESI): $m / z$ [M - H] $]^{-}$calcd for $\mathrm{C}_{15} \mathrm{H}_{15} \mathrm{ClN}_{3} \mathrm{O}_{4}$ : 336.0757; found: 336.0764 .

\section{$\mathrm{N}$-(6-Cyanopyridin-3-yl)-4-(2-methoxyethoxy)-1-methyl-6-oxo-} 1,6-dihydropyridine-3-carboxamide (10r)

From TMS-amine 9r. The precipitated product was washed with $\mathrm{MeCN} / \mathrm{Et}_{2} \mathrm{O}$ (1:1) to afford 10r as a pale yellow solid; yield: $220 \mathrm{mg}$ (67\%); $\mathrm{mp} 255-256^{\circ} \mathrm{C}$.

IR (ATR): 3312, 2225, 1684, 1648, 1580, $1540 \mathrm{~cm}^{-1}$.

${ }^{1} \mathrm{H}$ NMR (400 MHz, DMSO- $d_{6}$ ): $\delta=10.22(\mathrm{~s}, 1 \mathrm{H}$ ), $8.87(\mathrm{~d}, J=2.1 \mathrm{~Hz}, 1$ H), $8.43(\mathrm{~s}, 1 \mathrm{H}), 8.36(\mathrm{dd}, J=2.3,8.6 \mathrm{~Hz}, 1 \mathrm{H}), 8.05(\mathrm{~d}, J=8.7 \mathrm{~Hz}, 1 \mathrm{H})$, $6.01(\mathrm{~s}, 1 \mathrm{H}), 4.27(\mathrm{~m}, 2 \mathrm{H}), 3.79(\mathrm{~m}, 2 \mathrm{H}), 3.47(\mathrm{~s}, 3 \mathrm{H})$.

${ }^{13} \mathrm{C}$ NMR $\left(100 \mathrm{MHz}\right.$, DMSO- $\left.d_{6}\right): \delta=163.2,162.7,161.9,145.4,142.3$, $138.5,129.8,126.3,126.2,117.7,105.5,96.3,69.4,68.0,58.0,36.5$.

HRMS (HESI): $m / z$ [M - H] $]^{-}$calcd for $\mathrm{C}_{16} \mathrm{H}_{15} \mathrm{~N}_{4} \mathrm{O}_{4}$ : 327.1099; found: 327.1106 .

\section{Acknowledgment}

This work was generously supported by grants from the Canadian Institutes of Health Research (CIHR) (and HOP90183 and HOP120238).

\section{Supporting Information}

Supporting information for this article is available online at http://dx.doi.org/10.1055/s-0036-1588603.

\section{References}

(1) (a) Cheung, P. K.; Horhant, D.; Bandy, L. E.; Zamiri, M.; Rabea, S. M.; Karagiosov, S. K.; Matloobi, M.; McArthur, S.; Harrigan, P. R.; Chabot, B.; Grierson, D. S. J. Med. Chem. 2016, 59, 1869. (b) Grierson, D. S.; Cheung, P. K.; Chabot, B.; Harrigan, P. R.; Cochrane, A. W. PCT Int. Appl WO 2015164956, 2015.

(2) (a) Montalbetti, C. A. G. N.; Falque, V. Tetrahedron 2005, 61, 10827. (b) El-Faham, A.; Albericio, F. Chem. Rev. 2011, 111, 6557. (c) Humphrey, J. M.; Chamberlin, R. A. Chem. Rev. 1997, 97, 2243.

(3) Quelever, G.; Burlet, S.; Garino, C.; Pietrancosta, N.; Laras, Y.; Kraus, J-L. J. Comb. Chem. 2004, 6, 695.

(4) Dehe, D.; Munstein, I.; Reis, A.; Theil, W. R. J. Org. Chem. 2011, $76,1151$.

(5) (a) Lippert, J. W. III ARKIVOC 2005, (xiv), 87. (b) Carpino, L. A.; Beyermann, M.; Wenschuh, H.; Bienert, M. Acc. Chem. Res. 1996, 29, 268. (c) Carpino, L. A.; Sadat-Aalaee, D.; Chao, H. G.; DeSelms, R. H. J. Am. Chem. Soc. 1990, 112, 9651.

(6) (a) Rajeswari, S.; Jones, R. J.; Cava, M. P. Tetrahedron Lett. 1987, 28, 5099. (b) Wenschuh, H.; Bayermann, M.; Winter, R.; Bienert, M.; Ionescu, D.; Carpino, L. A. Tetrahedron Lett. 1996, 37, 5483. (c) Kim, H-O.; Gardner, B.; Kahn, M. Tetrahedron Lett. 1995, 36, 6013. (d) For a related reaction, see: Sakamoto, K.; Nakahara, Y.; Ito, Y. Tetrahedron Lett. 2002, 43, 1515.

(7) Wallace, E. M.; Lyssikatos, J.; Blake, J. F.; Seo, J.; Yang, H. W.; Yeh, T. C.; Perrier, M.; Jarski, H.; Marsh, V.; Poch, G.; Livingston, M. G.; Otten, J.; Hingorami, G.; Woessner, R.; Lee, P.; Winkler, J.; Koch, K. J. Med. Chem. 2006, 49, 441.

(8) (a) Carpino, L. A.; El-Faham, A. J. Am. Chem. Soc. 1995, 117, 5401. (b) El-Fahm, A.; Abdul-Ghani, M. Org. Prep. Proced. Int. 2003, 35, 369.

(9) (a) Joullié, M. M.; Lassen, K. M. ARKIVOC 2010, (viii), 189. (b) Carpino, L. A.; Ionescu, D.; El-Faham, A.; Beyermann, M.; Henklein, P.; Hanay, C. W. H.; Bienert, M. Org. Lett. 2003, 5, 975.

(10) Mai, K.; Patil, G. J. Org. Chem. 1986, 51, 3545.

(11) Spartan Version 6.1.7; Wavefunction Inc: Irvine (CA, USA), 2014.

(12) Sircar, J. C.; Capiris, T.; Bobovski, T. P.; Schwender, C. F. J. Org. Chem. 1985, 50, 5723.

(13) (a) Bryce, M. R.; Heaton, J. N.; Taylor, P. C.; Anderson, M. J. Chem. Soc., Perkin Trans. 1 1994, 1935. (b) El-Tamany, E-S. H.; Sowellim, S. Z.; Hamed, A. A.; Radwan, A. S. Res. Chem. Intermed. 2015, 41, 2675. (c) El-Tammany, E-S. H.; Hamed, A. A.; Sowellim, S. Z. A.; Radwan, A. S. Natural Sci. 2012, 4, 1013. (d) Schmidt, U.; Schwochau, M. Monatsh. Chem. 1967, 98, 1492.

(14) Fuchter, M. J.; Smith, C. J.; Tsang, M. W. S.; Boyer, A.; Saubern, S.; Ryan, J. H.; Holmes, A. B. Chem. Commun. 2008, 2152.

(15) Kricheldorf, H. R. Justus Liebigs Ann. Chem. 1971, 745, 81.

(16) (a) Garcia-Alvarez, R.; Crochet, P.; Cadierno, V. Green Chem. 2013, 15, 46. (b) Gernignon, N.; Al-Zoubi, R. M.; Hall, D. G. J. Org. Chem. 2012, 77, 8386. (c) Pattabiraman, V. R.; Bode, J. W. Nature 2011, 480, 471. 